\title{
Mudanças em comportamentos relacionados com o uso da voz após intervenção fonoaudiológica junto a educadoras de creche $^{* * *}$
}

\author{
Changes in behavior associated to the use of voice after a speech \\ therapy intervention with professionals of child day care centers
}

\author{
Marcia Simões-Zenari* \\ Maria do Rosário Dias de Oliveira Latorre**
}

*Fonoaudióloga. Doutora em Saúde Pública pela Faculdade de Saúde Pública da Universidade de São Paulo. Fonoaudióloga do Curso de Fonoaudiologia do Departamento de Fisioterapia, Fonoaudiologia e Terapia Ocupacional da Faculdade de Medicina da Universidade de São Paulo. Endereço para correspondência: Rua Prof. Wlademir Pereira, 61 - casa 3 - São Paulo - SP - CEP 05386-360 (marciasz@usp.br).

**Bacharel em Estatística. Professora Titular do Departamento de Epidemiologia da Faculdade de Saúde Pública da Universidade de São Paulo.

***Trabalho Realizado na Faculdade de Saúde Pública da Universidade de São Paulo e Curso de Fonoaudiologia da Faculdade de Medicina da

Universidade de São Paulo.

Artigo Original de Pesquisa

Artigo Submetido a Avaliação por Pares

Conflito de Interesse: não

Recebido em 02.06.2006. Revisado em 12.12.2006; 03.01.2008; 13.02.2008.

Aceito para Publicação em 13.02.2008.

\begin{abstract}
Background: preventive programs have been used with teachers with the purpose of lowering the number of risk factors for voice disorders. However, few studies have focused on the effectiveness of these programs. Aim: to assess changes in behaviors that are considered, according to the specific literature, as having a negative impact on the voice, during a speech therapy intervention program offered to educators. Method: an experimental study where a theoretical-practical program involving the professional use of voice was developed with 26 educators from two day care centers of São Paulo. This program involved five monthly meetings, working up to a total of twelve hours. During the program, topics concerning negative behaviors that affect the voice were discussed. On four of the meetings the educators filled in a protocol, making it possible to analyze the frequency on which they presented those specific behaviors. Later on, scores were given to the answers on the protocol in order to compare the findings during the program. For this comparison the statistical test of Wilcoxon and the linear tendency chi-square test were used. Results: the gradual decrease in the use of voice out of work was observed, as well as in speaking with a very low or high pitch voice and in eating excessively before sleeping. There was an increase in the occurrence of coughing in the third application of the protocol. As for the overall monthly averages, statistically significant differences were not found when comparing the four applications of the protocol. The overall average score was of 11.75, which was considered moderately abusive. Conclusion: the changes that were observed during the program were interesting, but very restrict which makes one wonder about the real effects of this kind of practice and how much broader changes rely on small individual changes. Actions that involve information about vocal well-being exclusively should be revised.
\end{abstract} Key Words: Voice; Voice Disorders; Program evaluation; Child Day Care Centers.

\section{Resumo}

Tema: programas preventivos têm sido indicados para diminuir a ocorrência de fatores de risco para alteração de voz em professores, mas poucos estudos têm enfocado sua eficácia. Objetivo: avaliar mudanças em comportamentos considerados na literatura especializada como negativos para a voz, ao longo de programa de intervenção fonoaudiológica oferecido a educadoras. Método: estudo experimental onde foi desenvolvido programa teórico-prático abordando uso vocal profissional junto a 26 educadoras de duas creches paulistas. Ocorreram cinco encontros mensais num total de doze horas. Durante o programa foram abordadas questões sobre comportamentos negativos para o uso vocal. As educadoras preencheram, em quatro encontros, protocolo que possibilitou analisar a freqüência em que apresentaram esses comportamentos. Após, foram construídos escores que possibilitaram comparar esses achados ao longo do programa, com uso dos testes de Wilcoxon e Qui-quadrado de tendência linear. Resultados: observouse diminuição gradativa no uso da voz fora do trabalho, no falar muito grave ou agudo e no comer em excesso antes de dormir. Houve aumento da ocorrência de tosse na terceira aplicação do protocolo. Quanto às médias gerais mensais, não foram observadas diferenças estatisticamente significativas ao se comparar as quatro aplicações. O escore médio geral foi 11,75, considerado moderadamente abusivo. Conclusão: as mudanças observadas ao longo do programa foram interessantes, mas muito restritas, levando à reflexão sobre o alcance deste tipo de prática e o quanto mudanças mais amplas não dependem apenas de pequenas mudanças individuais, o que em geral é preconizado. Ações que envolvam exclusivamente informações sobre bem estar vocal devem ser revistas.

Palavras-Chave: Voz; Distúrbios da Voz; Avaliação de Programas; Creches.

Referenciar este material como:

$\Omega$ Simões-Zenari M, Latorre MRDO. Mudanças em comportamentos relacionados com o uso da voz após intervenção fonoaudiológica junto a educadoras de creche. Pró$\sum 3$ Fono Revista de Atualização Científica. 2008 jan-mar;20(1):61-6. 


\section{Introdução}

As alterações vocais, mesmo em indivíduos que fazem uso profissional da voz, sempre foram vistas como questões individuais de saúde e não como alterações possivelmente relacionadas ao trabalho, o que dificulta a garantia por tratamento e a implantação de programas preventivos.

Dentre as diversas categorias profissionais, os professores apresentam alta ocorrência de problemas vocais, numa variação de $20 \%$ a $89 \%{ }^{(1)}$. Professores apresentam a voz mais alterada, referem mais sintomas vocais negativos, acreditam ter maior limitação no trabalho, reduzem suas atividades sociais por causa da voz e consideram com maior freqüência mudanças de ocupação devido à voz ${ }^{(2-3)}$. Educadores de creches se configuram como um grupo pouco estudado, apesar da alta prevalência de alteração vocal encontrada junto a eles ${ }^{(4-5)}$.

Uma vez detectada elevada ocorrência de problemas vocais entre professores, o que se indica é a necessidade de ações para minimizar o problema. Grillo e Penteado ${ }^{(6)}$ destacam que essas ações deveriam começar no período de formação do professor e se estender por toda a carreira. O que também é justificado pelo fato que professores no começo da carreira têm relatado maior número de sintomas vocais negativos, segundo estudos recentes ${ }^{(7-8)}$.

Por acreditar que o que falta ao professor é principalmente informação para o uso adequado da voz, tem sido uma prática comum do fonoaudiólogo ministrar palestras em instituições de ensino, abordando principalmente aspectos relativos ao bem estar vocal. Há poucos dados objetivos que avaliem este tipo de prática, ainda que sejam muitos populares ${ }^{(9-10)}$. O que se tem observado é que quando são utilizadas estratégias apenas informativas os resultados são mais limitados do que quando se agregam estratégias informativas e práticas ${ }^{(7,9,11-13)}$.

Com base no que foi exposto, o objetivo deste estudo foi avaliar mudanças em comportamentos consideradas negativos para o uso profissional da voz de educadoras de creche ao longo de um programa de intervenção fonoaudiológica teóricoprático voltado ao uso adequado da voz.

\section{Método}

Este estudo experimental foi aprovado pelo Comitê de Ética em Pesquisa da Faculdade de Saúde Pública da Universidade de São Paulo (protocolo 989/03) e todas as participantes assinaram termo de consentimento livre e esclarecido.
Participaram 26 educadoras, com idades entre 20 e 48 anos (média de 32,9 anos, desvio padrão = 7,5), de duas creches localizadas na região oeste da cidade de São Paulo, representando $100 \%$ da população inicial disponível. Essas creches foram selecionadas para este trabalho por terem participado de um estudo mais amplo envolvendo o uso vocal profissional, no qual constituíram o grupo experimental.

O programa de intervenção teórico-prático para o uso da voz do qual participaram teve um encontro inicial com quatro horas de duração e quatro encontros mensais de seguimento com duas horas cada, num total de 12 horas. Este formato foi estabelecido em conjunto com as equipes de coordenação das creches, a partir das suas rotinas e disponibilidades.

A base teórica foi amplamente trabalhada no primeiro encontro, sendo todos os conteúdos retomados nos encontros de seguimento, sempre de maneira inter-relacionada às práticas que estavam sendo propostas. Os tópicos trabalhados foram: processos de comunicação, produção da voz, psicodinâmica vocal, conceito de voz normal / voz adaptada / voz alterada, resistência e plasticidade vocal, comunicação não-verbal, corpo e voz, importância do ouvir, principais distúrbios vocais que acometem os professores, possibilidades vocais / limites individuais, conceito de bem estar vocal, principais fatores positivos e negativos para a voz do professor, importância do espaço físico no uso adequado da voz, técnicas de favorecimento para projeção vocal, ressonância, respiração, articulação dos sons da fala, resistência vocal, vibração das pregas vocais e alongamento cervical.

Nos encontros de seguimento foi solicitado às educadoras o preenchimento do Protocolo de Registro dos Comportamentos Relacionados ao Uso da Voz, traduzido e adaptado de Chan ${ }^{(14)}$. A tradução do protocolo foi feita por profissional habilitado e a adaptação se refere à maneira como foi aplicado, uma vez que no estudo original as professoras deveriam preencher o protocolo todos os dias e na presente pesquisa as educadoras foram convidadas a fazer isso mensalmente, como um recordatório da semana em que ocorria cada encontro. Deveriam indicar para cada um dos catorze comportamentos relacionados ao uso da voz listados no protocolo: "não fiz", "fiz um pouco" ou "fiz muito". Após o preenchimento as respostas foram consideradas como $0,1 \mathrm{e} 2$, respectivamente, para construção de um escore. A partir desse escore, que poderia variar de 0 a 28 , foi feita análise das mudanças dos comportamentos ao longo do 
programa. Esses escores foram ainda divididos em quartis para que se pudesse classificar os comportamentos em abusivo discreto (escores entre 1 e 7,7), moderado (entre 7,8 e 14,5), severo (entre 14,6 e 21,3) e extremo (entre 21,4 e 28).

Foi utilizado o teste de Wilcoxon para comparar as médias dos escores de cada comportamento e as médias totais, com as médias obtidas na primeira aplicação. Também foi utilizado o teste Qui-quadrado de tendência linear para analisar a tendência linear das médias de cada comportamento ao longo dos quatro encontros. Para as análises foram utilizados os softwares Epi Info, versão 6.0, SPSS, versão 12.0 para Windows e MedCalc, versão 7.2.0.2 para Windows. O nível de significância adotado foi de $5 \%$.

\section{Resultados}

Na Tabela 1 encontra-se a estatística descritiva dos escores dos comportamentos relacionados ao uso da voz e a comparação entre as quatro aplicações. A média geral obtida a partir dos dados dos quatro encontros foi 11,75 , o que se encaixa na classificação de comportamento moderadamente abusivo. Observa-se que não houve diferença estatisticamente significativa nas médias totais dos escores obtidos a partir da primeira aplicação do protocolo em comparação às demais, que ocorreram nos encontros mensais subseqüentes.

Na Tabela 2 estão apresentadas as médias de cada comportamento obtidas nas quatro aplicações e a médias gerais, assim como a análise estatística da tendência linear.

TABELA 1. Estatística descritiva dos escores dos comportamentos relacionados com o uso da voz cujos dados foram coletados a cada encontro de seguimento.

\begin{tabular}{c|c|c|c|c}
\hline Parâmetros & $\begin{array}{c}\text { Primeira } \\
\text { Aplicação }\end{array}$ & $\begin{array}{c}\text { Segunda } \\
\text { Aplicação }\end{array}$ & $\begin{array}{c}\text { Terceira } \\
\text { Aplicação }\end{array}$ & $\begin{array}{c}\text { Quarta } \\
\text { Aplicação }\end{array}$ \\
\hline média (dp) & $12,2(3,6)$ & $11,7(3,5)$ & $12,1(3,7)$ & $11,0(3,9)$ \\
mín-máx & $6-20$ & $5-19$ & $6-19$ & $4-19$ \\
mediana & 12,0 & 12,0 & 12,0 & 10,5 \\
p (Wilcoxon) & & 0,766 & 0,688 & 0,205 \\
\hline
\end{tabular}

TABELA 2. Distribuição das médias de cada comportamento relacionado ao uso da voz, obtidas ao longo dos quatro encontros de seguimento, e média geral.

\begin{tabular}{|c|c|c|c|c|c|c|}
\hline \multirow[b]{2}{*}{ Comportamentos } & \multicolumn{6}{|c|}{ Média } \\
\hline & Média Geral & $\begin{array}{c}\text { Primeira } \\
\text { Aplicação }\end{array}$ & $\begin{array}{c}\text { Segunda } \\
\text { Aplicação }\end{array}$ & $\begin{array}{c}\text { Terceira } \\
\text { Aplicação }\end{array}$ & $\begin{array}{c}\text { Quarta } \\
\text { Aplicação }\end{array}$ & $\mathrm{p}$ (Trend) \\
\hline usar a voz em recreação & 1,41 & 1,35 & $1,64(0,052)^{*}$ & $1,50(0,305)^{*}$ & $1,13(0,134) *$ & 0,092 \\
\hline falar alto no ruído & 1,36 & 1,35 & $1,40(0,714)^{*}$ & $1,42(0,564)^{*}$ & $1,25(0,796)^{*}$ & 0,134 \\
\hline falar muito rápido & 1,06 & 1,12 & $1,08(1,000)^{*}$ & $1,04(0,593)^{*}$ & $1,00(0,593)^{*}$ & 0,126 \\
\hline comidas irritativas & 0,99 & 1,15 & $0,96(0,206)^{*}$ & $0,88(0,088)^{*}$ & $0,96(0,166)^{*}$ & 0,112 \\
\hline bebidas irritativas & 0,96 & 0,96 & $0,88(0,414)^{*}$ & $1,08(0,317)^{*}$ & $0,91(0,414)^{*}$ & 0,164 \\
\hline falar muito alto ou baixo & 0,90 & 0,85 & $1,12(0,080)^{*}$ & $0,85(1,000)^{*}$ & $0,78(0,414)^{*}$ & 0,100 \\
\hline usar a voz fora do trabalho & 0,85 & 1,12 & $0,96(0,527)^{*}$ & $0,69(0,045)^{*}$ & $0,63(0,012)^{*}$ & 0,033 \\
\hline pigarrear & 0,79 & 0,77 & $0,56(0,331)^{*}$ & $1,04(0,118)^{*}$ & $0,79(0,782)^{*}$ & 0,244 \\
\hline chorar ou rir demais & 0,71 & 0,69 & $0,68(0,763)^{*}$ & $0,77(0,617)^{*}$ & $0,71(1,000)^{*}$ & 0,185 \\
\hline comer antes de dormir & 0,70 & 0,81 & $0,60(0,014)^{*}$ & $0,65(0,285)^{*}$ & $0,75(0,477)^{*}$ & 0,163 \\
\hline falar muito agudo ou grave & 0,69 & 0,85 & $0,72(0,439)^{*}$ & $0,69(0,285)^{*}$ & $0,50(0,005)^{*}$ & 0,049 \\
\hline falar muito emocionada & 0,64 & 0,77 & $0,60(0,218)^{*}$ & $0,58(0,342)^{*}$ & $0,61(0,194)^{*}$ & 0,107 \\
\hline tossir & 0,50 & 0,27 & $0,32(0,180)^{*}$ & $0,81(0,022)^{*}$ & $0,58(0,053)^{*}$ & 0,660 \\
\hline fumar & 0,16 & 0,19 & $0,16(0,317)^{*}$ & $0,12(0,157)^{*}$ & $0,17(1,000)^{*}$ & 0,145 \\
\hline
\end{tabular}

* p (Wilcoxon) 
Pode-se notar que a média geral mais baixa foi quanto ao fumar e a mais alta foi usar muito a voz em atividades de recreação. A partir da análise estatística realizada observou-se diminuição significativa em alguns comportamentos: usar a voz fora do trabalho, que apresentou menor ocorrência nos dois últimos encontros de seguimento, falar muito grave ou agudo, que diminuiu no último encontro e comer em excesso antes de dormir, que diminuiu apenas no terceiro encontro (segunda aplicação). Observouse aumento da tosse no quarto encontro (terceira aplicação) seguida de diminuição.

Houve tendência linear de diminuição estatisticamente significativa quanto ao uso da voz fora do trabalho e falar muito agudo ou grave. Nos demais comportamentos analisados não houve tendência linear estatisticamente significativa.

\section{Discussão}

O objetivo deste estudo foi avaliar mudanças em comportamentos consideradas negativos para o uso profissional da voz de educadoras ao longo de um programa de intervenção fonoaudiológica voltado ao uso adequado da voz.

$\mathrm{O}$ que se observou foi que a frequiência com que as educadoras participantes relataram os comportamentos investigados ao longo de quatro dos cinco encontros se manteve a mesma. Como a média geral apontou para um comportamento que foi classificado como moderadamente abusivo, isto pode significar dificuldade para diminuição, uma vez que podem estar próximas aos limites impostos por sua situação de vida e de trabalho. Se o comportamento geral médio tivesse sido classificado como severo ou extremo, poderia haver muito mais o que mudar. Um dado que pode reforçar esta hipótese é que os comportamentos isolados que apresentaram maior ocorrência foram usar muito a voz em atividades de recreação e falar alto no ruído, que acabam sendo mais inerentes à profissão de educador de crianças pequenas do que fumar ou tossir que foram os comportamentos relatados com menor frequiência.

Estudo realizado por Chan (14) encontrou redução no uso da voz e melhora das medidas vocais acústicas após o programa de intervenção. As professoras passaram a apresentar estratégias menos vocais para controlar as crianças que não o grito, o falar alto ou na presença do ruído. Para Grillo ${ }^{(15)}$ e Souza et al. (16) as professoras demonstraram dificuldades com as mudanças de hábitos e foram se esquecendo, ao longo do tempo, dos conteúdos trabalhados.
Yun et al. ${ }^{(17)}$ realizaram uma pesquisa com pacientes com pólipos que foram submetidos a um programa de três meses abordando o bem estar vocal, enquanto aguardavam a cirurgia de remoção. Após a conclusão do programa observou-se importante melhora na qualidade vocal dos participantes, sendo que para $38 \%$ deles a cirurgia não era mais indicada. Os pacientes que não fumavam ou que apresentavam pólipos pequenos foram os que mais se beneficiaram. Ainda assim, não observaram, entre o grupo que melhorou e o que não melhorou, diferenças significativas na diminuição de comportamentos como pigarrear, gritar, falar muito, beber mais água e em hábitos de alimentação.

Os três comportamentos isolados que ficaram menos freqüentes durante o programa analisado nesta pesquisa foram bastante comentados durante os encontros, devido ao grande interesse das educadoras. O uso da $\mathrm{voz}$ em excesso fora do trabalho foi discutido quando se tratou da importância dos momentos de repouso vocal e as participantes puderam perceber que há muitas possibilidades para se criar pequenos desses momentos ao longo do dia, sem que haja prejuízos para sua vida social. Södersten et al. ${ }^{(18)}$ indicam que um dos aspectos mais importantes de serem trabalhados junto a esta população é o aumento das pausas vocais entre as atividades.

O falar muito grave ou muito agudo foi repetidamente trabalhado nas práticas, por ser um aspecto que as incomodava e que facilmente percebiam, principalmente quando se tratou de psicodinâmica vocal e plasticidade vocal. Como indicado por Roy e Hendarto ${ }^{(19)}$, não se tratou aqui de um trabalho para obtenção do pitch ideal de cada indivíduo, o que é bastante questionado pela literatura, mas sim de adequação às atividades de trabalho desenvolvidas.

O comportamento de comer e logo em seguida dormir despertou o interesse delas na medida em que foi abordada sua forte correlação com o refluxo gastresofágico. Várias educadoras puderam identificar sintomas deste distúrbio e manifestaram vontade de procurar ajuda médica. Apesar do grande interesse pelo assunto, a diminuição deste comportamento foi isolada do segundo para o terceiro encontro, não se sustentando até o final do programa. A diminuição observada ocorreu principalmente entre aquelas educadoras que indicaram a prática freqüente deste comportamento. Muitas vezes hábitos que parecem simples de serem modificados fazem parte de uma rede mais complexa de fatores. Por outro lado, podem ter ocorrido 
mudanças mais qualitativas em relação a este comportamento, pois, na medida em que as educadoras não conseguiram mudar definitivamente seus horários de refeições, passaram a relatar a prática de refeições noturnas mais leves.

O aumento pontual na ocorrência de tosse no quarto encontro pode ser explicado pelo maior ocorrência de quadros gripais no mesmo período.

Bovo et al. ${ }^{(10)}$ utilizaram protocolo semelhante ao usado neste estudo, mas não descreveram especificamente os resultados relacionados às mudanças nos comportamentos. Indicaram que o uso deste instrumento, que no caso deles foi diário, preenchido de manhã, à tarde e à noite, pode ter sido útil como meio de motivação para que os professores diminuíssem a freqüência dos comportamentos abusivos, o que refletiu na melhora da qualidade vocal observada.

Muitas vezes os professores têm conhecimento sobre aspectos negativos para sua voz e nem por isso conseguem diminuí-los ou até mesmo eliminálos. Com freqüência, as tarefas e responsabilidades profissionais excessivas do dia-a-dia levam o educador a priorizar sua profissão, em detrimento de sua qualidade de vida. Isto se daria principalmente pelo fato de que esta profissão ainda é vista como uma missão, levando muitas vezes à dedicação irrestrita e justificando sacrifícios ${ }^{(20)}$.

Mesmo que os efeitos dos programas de intervenção se mostrem ainda restritos, parecem contribuir em algum grau para a melhora da voz e para a conscientização do educador sobre os comportamentos que apresenta e por que os apresenta $^{(7)}$.

Tavares e Martins (21) sugerem, além do desenvolvimento de programas preventivos junto aos professores, o controle dos fatores ambientais e das doenças associadas, o exame de laringe periódico e a garantia de acesso a adequado tratamento especializado em decorrência da alta prevalência de alterações laríngicas que têm sido encontradas nesta população.
É importante destacar que, ao se desenvolver um trabalho de intervenção dessa natureza, deve-se possibilitar o envolvimento de toda a comunidade escolar, para que não se fique centrado no campo das escolhas e opções individuais de estilos de vida de cada educador ${ }^{(20)}$. Dessa maneira, acredita-se que as mudanças podem ser mais amplas e duradouras. Se não houver mudança nas condições mais coletivas de trabalho, as ações, e conseqüentemente os resultados, sempre serão mais limitados ${ }^{(22)}$.

De modo geral as mudanças observadas neste trabalho foram interessantes, mas ainda em pequeno número, o que talvez fosse até esperado num programa de curta duração. A sistemática mensal foi a única possível e esta pode ter levado à desmotivação e esquecimento dos conteúdos. Programas com encontros mais próximos parecem levar a resultados mais expressivos ${ }^{(23)}$.

A principal questão a ser destacada a partir dos achados é a necessidade de mudanças coletivas e o quanto os fatores coletivos podem interferir nas decisões mais individuais, apontando que o foco deste tipo de programa não pode mesmo ser o indivíduo, mas a coletividade.

Os dados deste trabalho contribuem com evidências de que não é apenas a falta de informação que leva à ocorrência de alteração vocal em professores como acreditam Salas et al. ${ }^{(24)}$. Os achados apontam para um caminho muito mais complexo, onde é fundamental considerar todas as condições de trabalho. $\mathrm{O}$ apoio da legislação na área de saúde do trabalhador seria fundamental para proporcionar melhor uso da voz pelos educadores, assim como maior envolvimento das instituições onde estes trabalham ${ }^{(15,25)}$.

\section{Conclusão}

As mudanças observadas nos comportamentos relacionados à voz, após o programa de intervenção, foram: diminuição no uso da voz fora das atividades profissionais e no falar muito grave ou muito agudo. Houve diminuição seguida de aumento no hábito de se alimentar em horário próximo ao de dormir e houve aumento e depois diminuição na ocorrência de tosse. 


\section{Referências Bibliográficas}

1. Simões M, Latorre MRD. O. Alteração vocal em professores: uma revisão. Jornal Brasileiro de Fonoaudiologia. 2002;3(11):127-34.

2. Roy N, Merrill RM, Thibeault S, Gray SD, Smith EM. Voice disorders in teachers and the general population: effects on work performance, attendance, and future career choices. J. Speech Lang. Hear. Res. 2004;47:542-51.

3. Sliwinska-Kowalska M, Niebudek-Bogusz E, Fiszer M, Los-Spychalska T, Kotylo P, Sznurowska-Przygocka B, Modrzewska M. The prevalence and risk factors for occupational voice disorders in teachers. Folia Phoniatr. Logop. 2006;58(2):85-101.

4. Sala E, Laine A, Simberg S, Pentti J, Suonpää J. The prevalence of voice disorders among day care center teachers compared with nurses: a questionnaire and clinical study. J. Voice. 2001;15(3): 413-23.

5. Simões-Zenari M, Latorre MRDO. Prevalência de alteração vocal em educadoras de creche e sua relação com a auto-percepção. Rev Saúde Pública. 2006;40(6):1013-8.

6. Grillo MHMM, Penteado RZ. Impacto da voz na qualidade de vida de professore(a)s do ensino fundamental. Pró-Fono Revista de Atualização Científica, Barueri (SP). 2005;17(3):321-30

7. Ilomäki I, Mäki E, Laukkanen AM. Vocal symptoms among teachers with and without voice education. Logopedics Phoniatrics Vocology. 2005;30:171-4

8. Kooijman PGC, Thomas G, Graamans K, de Jong FICRS Psychosocial impact of the teacher's voice throughout the career. J. Voice. 2007;21(3):316-24.

9. Roy N, Weinrich B, Gray SD, Tanner K, Toledo SW, Dove H, Corbin-Lewis K, Stemple JC. Voice amplification versus vocal hygiene instruction for teachers with voice disorders: a treatment outcomes study. J. Speech Lang. Hear. Res. 2002;45:625-38

10. Bovo R, Galceran M, Petruccelli J, Hatzopoulos S. Vocal problems among teachers: evaluation of a preventive voice program. J. Voice. 2007;21(6):705-22

11. Roy N, Gray SD, Simon M, Dove H, Corbin-Lewis K, Stemple JC. An evaluation of the effects of two treatment approaches for teachers with voice disorders: a prospective randomized clinical trial. J. Speech Lang. Hear. Res. 2001;44:286-96.

12. Duffy OM, Hazlett DE. The impact of preventive voice care programs for training teachers: a longitudinal study. J. Voice. 2004;18(1):63-70.
13. Gillivan-Murphy P, Drinnan MJ, O'Dwyer TP, Ridha $\mathrm{H}$, Carding $\mathrm{P}$. The efectiveness of a voice treatment approach for teachers with self-reported voice problems. J. Voice. 2006;20(3):423-31.

14. Chan RW. Does the voice improve with vocal hygiene education? A study of some instrumental voice measures in a group of kindergarten teachers. J. Voice. 1994;8:279-91.

15. Grillo MHMM. The impact of a vocal improvement course in a speech language and hearing science prevent context. Pró-Fono Revista de Atualização Científica, Barueri (SP). 2004;16(2):159-68.

16. Souza TMT, Manzoni CRCT, Cunha PF, Clemente F. "A voz é meu instrumento": campanha de sensibilização vocal para educadores do município de São Paulo. Rev Soc Bras Fonoaudiologia, supl. esp. 2004:278.

17. Yun YS, Kim MB, Son Y. I. The effect of vocal hygiene education for patients with vocal polyp. OtolaryngologyHead and Neck Surgery. 2007;137:569-75.

18. Södersten M, Granqvist S, Hammarberg B, Szabo A. Vocal behavior and vocal loading factors for preschool teachers at work studied with binaural DAT recordings. J. Voice. 2002;16(3):356-71.

19. Roy N, Hendarto H. Revisiting the pitch controversy: changes in speaking fundamental frequency (SFF) after management of functional dysphonia. J. Voice. 2005;19(4):582-91.

20. Penteado RZ. Relações entre saúde e trabalho docente: percepções de professores sobre saúde vocal. Rev Soc Bras Fonoaudiol. 2007;12(1):18-22.

21. Tavares ELM, Martins RHG. Vocal evaluation in teachers with or without symptoms. J. Voice. 2007;21(4):407-14.

22. Giannini SPP, Passos MC. Histórias que fazem sentidos: as determinações das alterações vocais do professor. Distúrb Comum. 2006;18(2):245-57.

23. Simberg S, Sala E, Tuomainem J, Sellman J, Rönnema AA-M. The effectiveness of group therapy for students with mild voice disorders: a controlled clinical trial. J. Voice. 2006;20(1):97-109.

24. Salas SWA, Centeno HJ, Landa CE, Amaya CJM, Benites GMR. Prevalencia de disfonia en profesores del distrito de Pampas, Tayacaja, Huancavelica. Rev. Med. Hered. 2004;15(3):125-30.

25. Vilkman E. Voice problems at work: a challenge for occupational safety and health arrangement. Folia Phoniatr. Logop. 2000;52:120-5. 